\title{
Data Access
}

We have used German administrative data made available by the German Federal Employment Agency (Bundestanstalt für Arbeit) and the Institute for Employment Research (Institut für Arbeitsmarkt- und Berufsforschung). This data is confidential and cannot be made available as public use data set. The data and programs used for the paper can be accessed by interested researchers at the research data center of the Federal Employment Agency (http://fdz.iab.de). Please contact: iab.fdz@iab.de or stefan.bender@iab.de for further details. 